\title{
Scanning Transmission Ion Microscopy and Diffraction Imaging
}

John Notte IV, Raymond Hill, ${ }^{*}$ Shawn M McVey, Ranjan Ramachandra, ${ }_{* * * * * *}^{* *}$ Brendan Griffin, ${ }^{* *, * * *}$ David Joy ${ }^{* * * * * * *}$

* Carl Zeiss SMT Inc, One Corporation Way, Peabody, MA 01960, USA

*** Center for NanoPhase Materials Science, Oak Ridge National Laboratory, Oak Ridge, TN 37831-6496

*** Centre for Microscopy, Characterization and Analysis (M010), University of Western Australia, Crawley, WA 6009, Australia

*****Electron Microscopy Facility, University of Tennessee, Knoxville, TN 37996-0840

In this paper we investigate the possibility of applying the "ORION" (ZeissSMT : Peabody, MA) Helium ion scanning microscope (HIM) to imaging in the scanning transmission mode of operation. In particular because the interaction of $\mathrm{He}+$ ions with solids differs in many ways from that for electrons it is necessary to determine the changes in operating conditions, and in image interpretation, that may be required.

An important first question is what constitutes a 'thin' sample for a beam of 30-50keV He+ ions? Monte Carlo simulations of $\mathrm{He}+$ ion transport through thin foils show that for a given energy the signal falling on to an axial bright field (BF) detector remains close to $100 \%$ transmission for a range of thickness but then rapidly falls as plural scattering begins to dominate. Choosing that critical thickness as an estimate of the maximum usable range for transmission imaging, then (figure 1) for $40 \mathrm{keV}$ operation useful foils would be $250 \mathrm{~nm}$ thick for carbon, $150 \mathrm{~nm}$ for silicon, $50 \mathrm{~nm}$ for silver and $25 \mathrm{~nm}$ for gold. At $100 \mathrm{keV}$ these value are about 2 to 3 times higher. These calculations do not take into account the enhanced transmission (channeling) of ions in crystals and so are likely an underestimate of the performance. Monte Carlo simulations to determine the broadening of the ion beam at the exit surface of the specimen were also made (figure 2). For silicon at 40keV the Full Width at Half Maximum height (FWHM) of the beam, assuming an incident probe of zero diameter, is less than $3 \mathrm{~nm}$ for all thicknesses up to $150 \mathrm{~nm}$, although a skirt of scattered ions that gradually increase in both intensity and width is visible but remains relatively insignificant. Similar results are predicted for other elements confirming that, within the constraints imposed on the usable sample thickness, scanning transmission ion microscopy will be capable of offering high resolution imaging.

In a STEM system detectors are typically provided for on axis Bright Field (BF) and the off axis High Angle Annular Dark Field (HAADF) observations. As predicted by Cowley's principle of reciprocity the acceptance angle of the $\mathrm{BF}$ detector has a significant effect on the appearance of the image. If the BF detector acceptance is confined to a small fraction of a Bragg angle then the BF STEM image will appear similar to a conventional TEM bright field image of the same material, but at the expense of poor signal to noise ratio. A larger BF detector will collect more signal, but the form of the image will increasingly deviate from the conventional TEM version. The annular HAADF detector has an inner radius chosen to be large enough (50 millirads or more) to exclude any Bragg scattered electrons and the collected signal is then solely a function of the specimen thickness and atomic number. The same considerations apply to the detectors for the $\mathrm{He}+\mathrm{STIM}$, but practical considerations intervene. For a $40 \mathrm{keV} \mathrm{He}+$ beam a typical Bragg 


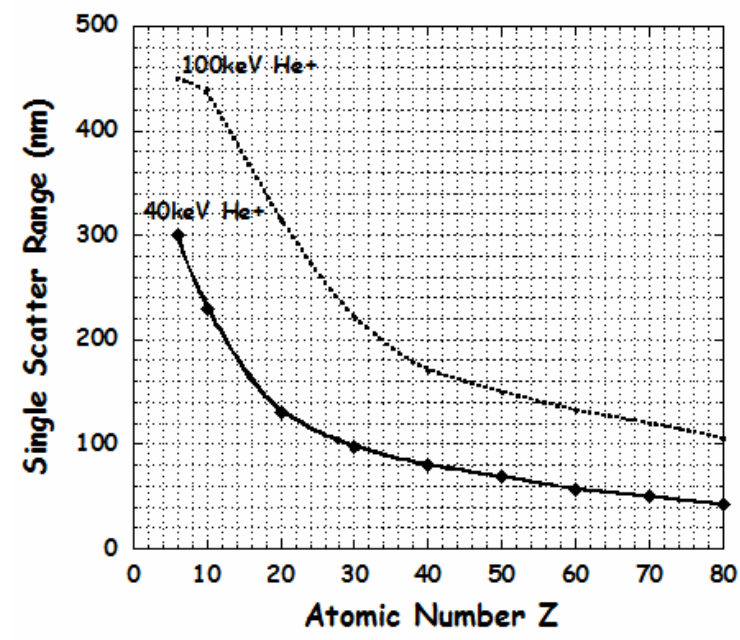

Fig.1 Computed single scatter range for $\mathrm{He}+\mathrm{vs}$ atomic number at 40 and $100 \mathrm{keV}$

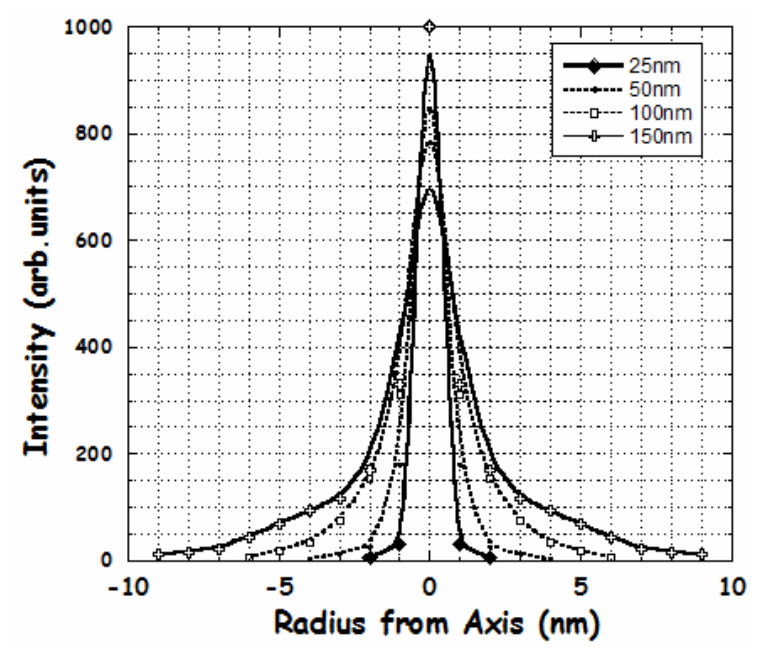

Fig.2 Computed probe profile of $40 \mathrm{keV} \mathrm{He}+$ beam in silicon vs thickness

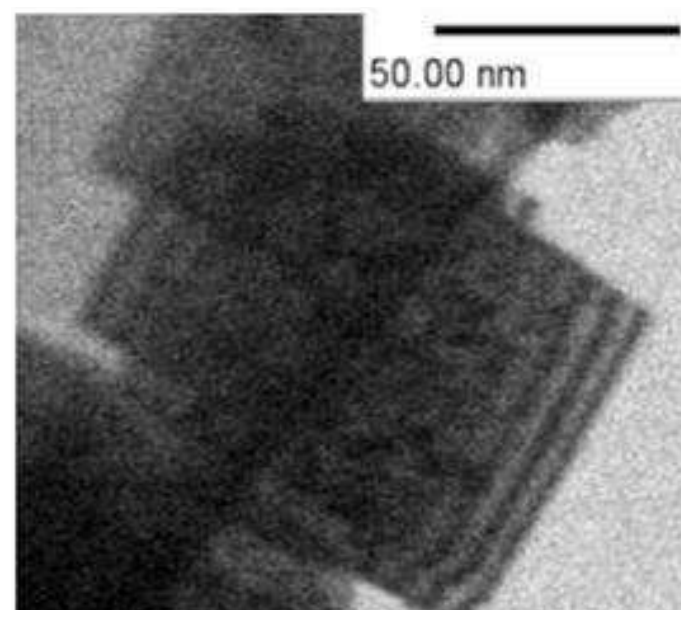

Fig.3 BF transmission image of $\mathrm{MgO}$ with $40 \mathrm{keV} \mathrm{He}+$ beam showing thickness fringes and defects

angle is about 0.2 millirads but with the arrangement used here the $\mathrm{BF}$ detector accepts ions from 0 to 10 mrads off axis, and the HAADF detector from 10 to 40 mrads. As a consequence the characteristics of both signal modes will differ from the ideal and show some symptoms of overlap. Using $\mathrm{MgO}$ crystals from 10 to $100 \mathrm{~nm}$ in size as samples, a beam energy of $40 \mathrm{keV}$, and a beam current of about $1 \mathrm{pA}$, excellent images were recorded in both $\mathrm{BF}$ or DF modes. The general appearance of the images showed the $\mathrm{BF}$ and $\mathrm{DF}$ to be generally complementary to each other with the BF optimizing sample topography and shape, and with the DF emphasizing difference in mass and composition. Line traces across the vertical edges of crystals confirmed that the probe limited resolution was of the order of 2-3nm as predicted by the simulations. The $\mathrm{MgO}$ cubes also produced contrast identifiable as being the result of ion diffraction. For example (figure 3 ) the BF signal from some wedge shaped regions yielded contrast clearly related to the thickness fringes observed in the TEM, and contrast from dislocations and grain boundaries was also clearly visible. Although the theory of ion diffraction is the same as for electrons there are practical differences. For example, He+ ions, because of their shorter wavelength, are represented by an Ewald sphere much larger than that for an electron beam of the same energy, in addition the need for thin $\mathrm{He}+$ samples results in an increased extension of the reciprocal lattice points in the z-direction. These two factors will greatly increase the number of Bragg beams that can be simultaneously excited making it difficult to achieve a simple two beam condition suitable for defect analysis. 